\title{
Frontières
}

\section{Mourir dans la dignité}

\section{La loi belge relative à l'euthanasie, une réponse légale}

\section{Jacqueline Herremans}

Volume 24, numéro 1-2, automne 2011, printemps 2012

L’aide médicale à mourir

URI : https://id.erudit.org/iderudit/1013087ar

DOI : https://doi.org/10.7202/1013087ar

Aller au sommaire du numéro

Éditeur(s)

Université du Québec à Montréal

ISSN

1916-0976 (numérique)

Découvrir la revue

Citer cet article

Herremans, J. (2011). Mourir dans la dignité : la loi belge relative à

l'euthanasie, une réponse légale. Frontières, 24(1-2), 73-80.

https://doi.org/10.7202/1013087ar d'utilisation que vous pouvez consulter en ligne.

https://apropos.erudit.org/fr/usagers/politique-dutilisation/ 


\section{MOURIR \\ DANS LA DIGNITÉ La loi belge relative à l'euthanasie, une réponse légale}

\begin{abstract}
Jacqueline Herremans, avocate au barreau de Bruxelles, membre du Comité consultatif de bioéthique, membre de la Commission fédérale belge de contrôle et d'évaluation de la loi relative à l'euthanasie.
\end{abstract}

En 2002, le législateur belge a adopté trois lois qui ont un impact incontestable sur le droit médical en général et sur les décisions médicales en fin de vie en particulier ${ }^{1}$. La loi sur les droits du patient, d'initiative gouvernementale, a consacré certains principes dégagés par la doctrine et la jurisprudence, notamment le respect de l'autonomie du patient, tout traitement médical devant faire l'objet du consentement éclairé du patient. La loi relative à l'euthanasie et celle relative aux soins palliatifs ont été initiées par le Parlement. La volonté des parlementaires a été de briser l'opposition entre soins palliatifs et euthanasie, en consacrant d'une part le principe de l'accès général aux soins palliatifs et en proposant d'autre part une dépénalisation conditionnelle de l'euthanasie.

Dans ce texte, j'envisagerai d'abord la situation antérieure à l'adoption de la loi sur le plan juridique et déontologique, ainsi que dans la société ; j'évoquerai ensuite les débats parlementaires qui ont précédé et entouré son adoption; enfin, je rendrai compte des éléments principaux de cette loi et de la modification du code de déontologie qu'elle a suscitée.

\section{SITUATION ANTÉRIEURE \\ SUR LE PLAN JURIDIQUE \\ ET DÉONTOLOGIQUE \\ ET DANS LA SOCIÉTÉ}

\section{CODE PÉNAL BELGE}

Avant l'adoption de la loi relative à l'euthanasie, le droit pénal belge, fondé sur le Code Napoléon, ne prévoyait pas d'incrimination spécifique pour l'acte qui consistait à mettre fin à la vie d'une personne à sa demande. Dès lors, si un médecin accédait à la demande d'un patient de mettre fin à sa vie, il était susceptible de devoir répondre de la charge d'homicide volontaire avec préméditation, soit d'assassinat, voire d'empoisonnement (article 392 et suivants du Code pénal). Aucune affaire n'a cependant débouché sur un procès public. Ceci s'explique d'une part par le principe de l'opportunité des poursuites qui relève du Parquet, autorité judiciaire poursuivante, et d'autre part par une construction jurisprudentielle basée sur la notion de force majeure, l'état de nécessité.

Le Ministère public belge est en effet libre d'estimer l'opportunité de traduire devant les tribunaux des personnes sur lesquelles reposent des charges d'infraction pénale. Il semblerait que pour les questions de fin de vie, les procureurs généraux ont préféré adopter une attitude prudente, redoutant sans doute les effets dans la société de tels procès, impliquant principalement des médecins. Leur attitude avait été tout autre pour le débat que la Belgique a connu antérieurement à propos de l'avortement: des poursuites ont alors été engagées à l'égard de femmes ayant subi des avortements, de médecins, et de paramédicaux. L'enjeu était cependant nettement différent dans ces cas de procès relatifs à des avortements. En effet, ceux-ci impliquaient d'autres personnes que les seuls médecins et les juridictions compétentes étaient les tribunaux correctionnels puisque l'on parlait de délit et non de crime. Les femmes qui faisaient l'objet de poursuites étaient en mesure de parler de leur vécu de leur détresse. Les peines qui ont été prononcées étaient assorties de sursis, même à l'égard de médecins. Et il faut souligner que les autorités judiciaires lançaient un appel clair à l'égard du monde politique: «En l'absence d'initiative législative, nous n'avons pas d'autre solution que d'appliquer la loi pénale et de réprimer l'avortement. » 
Aux Pays-Bas, l'attitude des autorités judiciaires a été tout autre avant l'adoption de la loi relative à l'euthanasie: des procès ont été engagés à l'égard de médecins ayant pratiqué une euthanasie. Alors que ce pays connaît également un système légal basé sur le Code Napoléon, les autorités judiciaires ont agi à la manière d'un pays appliquant la common law, et la jurisprudence a joué un rôle très important (voir Leleu et Genicot, 2004). Il faut préciser que le Code pénal néerlandais comporte des incriminations particulières pour l'interruption volontaire de la vie à la demande de la personne (soit l'euthanasie) et l'assistance au suicide, délits sanctionnés respectivement par des peines d'emprisonnement de maximum 12 et 3 ans ou d'une amende (articles 293 et 294 du Code pénal). Les médecins faisant l'objet de poursuites judiciaires pour de tels délits sont traduits devant les tribunaux correctionnels et ne sont donc pas soumis à la lourdeur et au poids émotionnel de la procédure devant la cour d'assises. Par ailleurs, aux PaysBas, la justice ne connaît pas les arriérés judiciaires endémiques de la Belgique et les médecins peuvent compter sur un procès dans des délais raisonnables.

La première affaire en cette matière date de 1973, le tribunal correctionnel de Leeuwaarden condamnant à une peine de principe (une peine de prison d'une semaine avec un sursis d'un an) la doctoresse Postma qui avait aidé sa mère à mourir à la demande répétée et insistante de celle-ci qui souffrait d'une maladie incurable lui causant des souffrances morales ou physiques intolérables. Une autre étape importante fut la décision du Hoge Raad (l'équivalent de la Cour de cassation en Belgique) du 27 novembre 1984 (affaire Schoonheim) aux termes de laquelle la Cour introduisit la notion d'excuse pour cause de force majeure pour justifier l'euthanasie pratiquée par un médecin dans le cas d'une demande d'une patiente présentant des souffrances insupportables (voir Leenen, 1985). La jurisprudence franchissait un pas décisif en considérant le conflit de valeurs auquel était confronté le médecin et en l'acquittant. À l'occasion de ces diverses affaires, elle affinait ainsi les critères de prudence à suivre par le médecin non seulement pour bénéficier d'un sursis mais aussi pour entendre son geste justifié. Un débat transversal eut lieu dans la société impliquant tant les professionnels de la santé, dont l'Académie royale de médecine, que les milieux académiques. À sa suite, les PaysBas ont adopté la loi du 12 avril 2001 dite de contrôle de l'euthanasie et du suicide assisté ${ }^{2}$, après avoir connu une dépénalisation de fait avec obligation pour les méde- cins de déclarer les cas d'euthanasie auprès de commissions régionales de contrôle.

En Belgique, des poursuites judiciaires furent certes engagées avant le vote de la loi de dépénalisation de l'euthanasie. Ce fut notamment le cas d'un médecin qui connut les affres d'une longue instruction dans l'arrondissement de Nivelles. Ce médecin plaida devant les juridictions d'instruction que, confronté à un conflit de valeurs, il s'était senti contraint de prendre le risque de transgresser la loi pour répondre à la demande de son patient de mettre fin à ses souffrances. Sur réquisitoire du ministère public, la Chambre du Conseil de Nivelles a suivi la thèse de l'état de nécessité et a prononcé un non-lieu en 1997. Il y eut également l'affaire de l'hôpital de la Citadelle à Liège qui a éclaté au début de l'année 2000. Deux médecins ont été inculpés, l'un d'entre eux fut arrêté et placé en détention préventive pendant cinq jours. Le 6 février 2003, la Chambre du Conseil de Liège a prononcé un non-lieu, se basant sur le respect par ces médecins des conditions essentielles de la loi relative à l'euthanasie entrée en vigueur le 22 septembre 2002 (voir Herremans, 2003).

\section{CODE DE DÉONTOLOGIE MÉDICALE}

En Belgique, le médecin était non seulement confronté à l'interdit pénal, mais aussi à l'interdit déontologique. Jusqu'en 1992, le Code de déontologie médicale ${ }^{3}$ prévoyait à l'article 95: «Provoquer délibérément la mort d'un malade, quelle qu'en soit la motivation, est un acte criminel », et précisait à l'article 96: «Cet acte ne trouve aucune justification dans le fait qu'il soit sollicité expressément par le malade.»

La réforme du 17 octobre 1992 a modifié ce texte de la manière suivante: article 95 :

Le médecin ne peut pas provoquer délibérément la mort d'un malade ni

l'aider à se suicider;

article 96:

Lorsqu'un malade se trouve dans la phase terminale de sa vie tout en ayant gardé un certain état de conscience, le médecin lui doit toute assistance morale et médicale pour soulager ses souffrances morales et physiques et préserver sa dignité. Lorsque le malade est définitivement inconscient, le médecin se limite à ne prodiguer que des soins de confort.

L'interdit de l'euthanasie était donc maintenu mais la notion de «dignité du malade» apparaissait. C'est dans le contexte de telles normes déontologiques que prit place le débat dans la société.
DÉBAT DANS LA SOCIÉTÉ ET LE RÔLE

DU COMITÉ CONSULTATIF DE BIOÉTHIQUE

Au sein de la société, le débat sur l'euthanasie, certes difficile, avait été initié notamment par des associations telles que l'Association pour le droit de mourir dans la dignité, - en abrégé ADMD -, présidée à l'époque par un médecin, Yvon Kenis, oncologue.

Le Comité consultatif de bioéthique joua également un rôle important. Le 12 mai 1997, il publiait son premier avis qui concernait l'opportunité d'un règlement légal de l'euthanasie. Le symbole était fort: la demande d'avis émanait des présidents de la Chambre des représentants et du Sénat et l'avis était le premier rendu depuis la constitution de ce comité 4 .

La demande d'avis en question allait au-delà de la seule « question de l'opportunité d'un règlement légal de l'interruption de vie à la demande des malades incurables ("euthanasie")", elle concernait aussi «les soins palliatifs, la déclaration de volonté relative au traitement et le "testament de vie" » ainsi que «les propositions de loi actuellement pendantes en la matière». Le Comité a toutefois préféré se limiter dans un premier temps à la question de l'opportunité d'une intervention législative en matière d'euthanasie pour les patients conscients. C'est dans un deuxième temps qu'il envisagea la question de l'arrêt actif de la vie des personnes incapables d'exprimer leur volonté (voir l'avis $n^{\circ} 9$ du 22 février 1999).

Une remarque sur la méthode de travail du Comité consultatif de bioéthique belge est nécessaire pour comprendre la teneur de ses avis. Ce comité ne travaille pas au consensus, mais veut rendre compte de façon argumentée de positions qui peuvent être défendues au sein de la société belge ; il insiste donc à la fois sur les points de consensus et de dissensus. Son premier avis relatif à l'euthanasie est un bel exemple de cette approche.

Le comité proposa une définition de l'euthanasie qui sera reprise dans la loi: "acte pratiqué par un tiers qui intentionnellement met fin à la vie d'une personne à la demande de celle-ci». Il reconnaissait l'existence, au sein de la société, de conceptions éthiques différentes et même irréductiblement divergentes. Certains estimaient que l'euthanasie est justifiée sur le plan moral lorsque se conjuguent une situation médicale sans issue, une souffrance intense et le droit élémentaire de tout homme à l'autonomie. Pour d'autres, la valeur intangible de la vie est un principe qui ne souffre pas d'exception. D'autres enfin ne tolèrent cette transgression qu'à titre exceptionnel, dans des 
situations extrêmes et au terme d'un débat éthique.

Ces conceptions éthiques divergentes devaient nécessairement déboucher sur des propositions différentes de règlement légal. Quatre propositions furent formulées. Une seule d'entre elles exigeait le statu quo, tandis que les trois autres reconnaissaient la nécessité d'une réglementation allant de la reconnaissance de l'état de nécessité avec contrôle $a$ priori à la dépénalisation de l'euthanasie sous conditions, en passant par la dépénalisation avec contrôle a posteriori. En voici une brève synthèse.

1. La proposition de modification législative dépénalisant l'euthanasie faisait référence au principe que la loi doit garantir explicitement le droit de tout individu de disposer lui-même de sa vie et de vivre selon ses convictions propres, dans le respect de celles des autres, dans la mesure où l'acte en cause ne constitue pas un danger potentiel pour autrui ou pour la société. Les tenants de cette position avançaient en outre qu'une dépénalisation de l'euthanasie garantirait par ailleurs au médecin un espace de sécurité juridique.

2 . Une régulation "procédurale» a posteriori de l'euthanasie décidée en colloque singulier s'inspirait du modèle des PaysBas qui connaissaient à l'époque une dépénalisation de fait. Cette proposition de procédure qui ne comportait pas de modification du Code pénal reposait sur le colloque singulier du malade avec son médecin. Le contrôle de la société se ferait a posteriori, le médecin étant tenu d'informer les autorités judiciaires, via le médecin légiste.

3. Une régulation "procédurale» $a$ priori des décisions médicales les plus importantes concernant la fin de vie, $y$ compris l'euthanasie, après consultation collégiale visait à élargir le cadre des décisions médicales en fin de vie et à impliquer non seulement le patient et le médecin, mais aussi l'équipe soignante, la famille et enfin un membre non-médecin du comité d'éthique local. Le médecin qui procéderait ensuite à une euthanasie se verrait couvert par la notion d'«état de nécessité».

4. Le maintien pur et simple de l'interdit légal de l'euthanasie exprimait la volonté de maintenir le statu quo, c'est-à -dire l'interdiction en toute circonstance de la pratique de l'euthanasie. Ceux qui soutenaient cette position la justifiaient notamment par un double refus: le refus absolu de transgresser l'interdit de tuer et le refus de s'engager dans la reconnaissance officielle d'un pouvoir, estimé "démesuré», de vie et de mort du médecin sur le patient.

L'avis concluait que l'examen de la troisième position avait permis un rapprochement entre plusieurs adversaires et partisans de l'euthanasie. Dans cette ouverture du dialogue, il faut souligner le rôle très important assumé, au sein de la commission restreinte chargée de préparer l'avis, par le professeur Fernand Van Neste, auteur, dans les pages qui suivent, de l'article concernant la Commission fédérale de contrôle et d'évaluation de la loi.

Sur base de ce premier avis du Comité consultatif de bioéthique, le Sénat, sous l'égide de Roger Lallemand ${ }^{5}$, organisa, les 9 et 10 décembre 1997, un débat sur l'euthanasie. Celui-ci se révéla fort ouvert et fructueux, et il montra l'intérêt des parlementaires pour ces questions de fin de vie. Un consensus se dégagea sur les points suivants: la définition de l'euthanasie, la nécessité de développer les soins palliatifs, la nécessité d'informer le patient, le respect de la clause de conscience pour le médecin et la nécessité de poursuivre le débat en commissions. Le seul parti politique qui s'opposa à l'ouverture de ce débat fut le Vlaams Blok, parti que l'on peut qualifier d'extrême droite. Le périodique du Sénat titra: "Euthanasie: Un dialogue au-delà des clivages politiques ».

\section{DÉBATS PARLEMENTAIRES}

\section{DES PROPOSITIONS DE LOI \\ ET DES AUDITIONS \\ LARGEMENT DIFFUSÉES}

Depuis 1984, des propositions de loi étaient régulièrement déposées afin de légiférer en cette matière. Mais elles ne franchissaient pas le cap nécessaire du renvoi en commissions. Le monde politique gardait certainement en mémoire le long et douloureux débat autour de la question de l'interruption volontaire de grossesse et la crise institutionnelle que suscita le vote de la loi du 3 avril 1990 qui y est relative. Non seulement une majorité de rechange (libéraux-socialistes) s'était formée au sein du Parlement pour adopter la proposition de loi Lallemand Herman Michielsens, mais, par sa lettre du 30 mars 1990, le roi Baudouin avait fait part au Premier ministre, à la tête d'une coalition formée par les sociaux-chrétiens et les socialistes, du "grave problème de conscience» auquel il était confronté: il craignait que la future loi n'apporte «une diminution du respect de la vie de ceux qui sont les plus faibles». Il refusait donc de sanctionner et de promulguer cette loi votée par le Parlement. Pour faire face à cette situation inédite, le roi fut déclaré dans l'impossibilité de régner, le temps nécessaire au Conseil des ministres pour procéder aux formalités de sanction et de promulgation de la loi.

L'une des conséquences immédiates de cette crise a été de rendre purement symbolique le dépôt de propositions de loi en matière de fin de vie par des parlementaires au cours des législatures suivantes. Or, depuis le milieu des années 1980, d'Edouard Klein à Hugo Coveliers, en passant par Philippe Monfils, Eddy Boutmans, Jo Cuyvers, Serge Moureaux, Frans Lozie et Daniel Bacquelaine, plusieurs parlementaires s'étaient essayés à cet exercice difficile de coucher sur papier les principes législatifs en matière de fin de vie. Il était cependant exclu que le Parlement s'attache à l'examen de ces propositions. En effet, les diverses déclarations gouvernementales comportaient un véto à l'égard de la prise en compte des problèmes éthiques, véto imposé par les partis catholiques échaudés par le vote de la loi relative à l'avortement grâce à une majorité alternative. Après le débat des 9 et 10 décembre 1997, les sénateurs Fred Erdman (socialiste flamand) et Roger Lallemand (socialiste francophone) déposèrent le 2 février 1999 une proposition de loi relative aux problèmes de la fin de vie et à la situation du patient incurable. Cette proposition de loi abordait tant la question du droit aux soins palliatifs que celle de l'euthanasie, les auteurs proposant d'insérer dans le Code pénal un article 417 bis prévoyant une cause de justification pour l'acte posé par un médecin dans le respect des conditions précisées par la loi. Initiative sans doute trop tardive pour que le débat parlementaire puisse reprendre avant la fin de la session législative, mais bien à propos afin que cette question puisse être débattue pendant la campagne électorale de juin 1999.

La déclaration gouvernementale $\mathrm{du}$ premier gouvernement Verhofstadt confiait au Parlement le soin de s'emparer des questions éthiques et en particulier de celles portant sur la fin de la vie. Ce gouvernement baptisé «arc-en-ciel» était composé de libéraux, de socialistes et d'écolos, les partis catholiques étant rejetés dans l'opposition.

Les propositions relatives aux questions de fin de vie furent relevées de caducité et, dès le 20 octobre 1999, les commissions de la justice et des affaires sociales du Sénat tenaient leur première réunion consacrée à l'examen de ces différents textes. Il s'agissait des propositions suivantes: proposition de loi relative aux problèmes de fin de la vie et à la situation du patient incurable (de M. Philippe Mahoux et Mme Myriam Vanlerberghe), proposition de loi 
relative à la demande d'interruption de vie (de M. Philippe Monfils) et proposition de loi élargissant le droit de codécision du patient par l'institution d'une déclaration de volonté relative au traitement (de M. Frans Lozie et Mme Jacinta De Roeck).

En décembre 1999, messieurs Philippe Mahoux, Philippe Monfils, et mesdames Jeannine Leduc, Myriam Vanlerberghe, Marie Nagy et Jacinta De Roeck, six sénateurs issus de partis de la majorité gouvernementale unirent leurs réflexions et déposèrent des propositions portant tant sur les soins palliatifs que sur l'euthanasie.

Vouloir retracer les travaux parlementaires exigerait le talent de Mozart pour l'air de Leporello dans Giovanni «Madamina, il catalogo » : en effet, plus de 40 auditions eurent lieu, qui furent généralement publiques, sauf exception demandée par certains témoins, et qui furent retransmises en direct et dans leur intégralité à la télévision belge; il y eut aussi plus de 86 réunions des commissions sénatoriales qui se sont traduites par 1358 pages, des centaines d'amendements, etc.

On ne soulignera jamais assez l'impact de ces auditions tant sur le monde politique que dans la société. Leur retransmission intégrale par la télévision belge, sauf celles qui se tenaient à huis clos à la demande de quelques rares professionnels de la santé, a permis de trouver un écho dans le public et dans les médias. Et les soins palliatifs ne furent certes pas absents du débat.

\section{L'AVIS DU CONSEIL D'ÉTAT}

\section{DU 2 JUILLET 2001}

L'avis du Conseil d'État du 2 juillet 2001 constitua une étape fondamentale, particulièrement en ce qui concerne la question de la conformité du texte proposé aux instruments internationaux protégeant les droits humains. Après une analyse fouillée de ce qui était encore une proposition de loi, le Conseil d'État se proposa favorablement sur la compatibilité des textes débattus avec les dispositions de la Convention européenne des droits de l'homme et du Pacte international relatif aux droits civils et politiques garantissant le respect de la vie qui doivent s'interpréter au regard du droit à l'autodétermination (voir Rigaux, 2002).

Rappelons que le droit à la vie est garanti notamment par l'article 2 de la Convention européenne des droits de l'homme (CEDH) : «Le droit de toute personne à la vie est protégé par la loi » et par l'article 6 du Pacte international relatif aux droits civils et politiques (PIDCP): «Le droit à la vie est inhérent à la personne humaine. Ce droit doit être protégé par la loi. » Le principe selon lequel le droit à la vie doit être protégé n'impose pas seulement une obligation négative à l'État, à savoir l'interdiction de priver un individu de la vie, mais également une obligation positive, à savoir l'obligation de prendre les mesures nécessaires à la protection de la vie des personnes relevant de sa juridiction. La question, dit le Conseil d'État, «est de savoir si, en instaurant une dépénalisation de l'euthanasie telle que la prévoit la proposition, le législateur manquerait à son devoir de protéger le droit à la vie».

Le Conseil d'État cite la jurisprudence de la Cour européenne des droits de l'homme aux termes de laquelle les articles 2 de la CEDH et 6 du PIDCP n'impliquent nullement une obligation des autorités de protéger «la vie» en tant que telle, contre le gré de l'individu. Il s'agit plutôt du devoir de protéger "le droit à la vie». Dès lors, il convient d'examiner dans quelle mesure le souhait de mourir d'un individu influence l'importance de l'obligation positive des autorités de protéger le droit à la vie.

Le Conseil d'État poursuit son analyse en soulignant que l'obligation des autorités doit être mise en balance notamment avec le droit à l'autodétermination de l'intéressé.

Du point de vue des droits

fondamentaux, cela signifie que

l'obligation des autorités de protéger

le droit à la vie (article 2 de la $\mathrm{CEDH}$

et article $6 \mathrm{du}$ PIDCP) doit être mise

en balance avec le droit de l'intéressé

d'être protégé contre les traitements

inhumains ou dégradants (article 3

de la CEDH et article 7 du PIDCP),

et avec son droit au respect de son

intégrité physique et morale, qui

relève du droit au respect de la vie

privée (article $8 \mathrm{CEDH}$ et article 17

PIDCP).

Le Conseil d'État note qu'«une des caractéristiques essentielles du débat sur l'euthanasie tient en ce qu'il suscite des questions éthiques difficiles et fondamentales, si bien qu'il s'impose de faire un choix entre différentes conceptions éthiques opposées». Pour lui, «L'appréciation du caractère légitime ou non d'une proposition de loi visant à dépénaliser l'euthanasie requiert également de partir du principe que c'est au législateur qu'il appartient de concilier des conceptions éthiques opposées. Le juge doit à cet égard respecter le pouvoir d'appréciation du législateur et ne peut se substituer à lui. »

Après avoir remarqué que la proposition ne touche en rien aux dispositions du Code pénal qui répriment l'assassinat, le meurtre ou la mort par imprévoyance ou négligence, et examiné les divers gardefous prévus par les parlementaires, le Conseil d'État conclut «que même si elle prévoit une limitation de la protection du droit à la vie accordée jusqu'à présent par la loi, la proposition de loi relative à l'euthanasie soumise pour avis reste dans les limites imposées à la marge d'appréciation de l'autorité nationale par les articles 2 de la $\mathrm{CEDH}$ et $6 \mathrm{du} \mathrm{PIDCP} »$.

\section{ÉLÉMENTS FONDAMENTAUX DE LA LOI DU 28 MAI 2002}

J'envisagerai ici les aspects principaux de la loi : la définition de l'euthanasie et les principes qui la fondent, les obligations énoncées et leurs implications, ainsi que la déclaration anticipée concernant les personnes inconscientes, le contrôle sociétal de la loi, le rôle des pharmaciens, la liberté de conscience, l'assimilation à la mort naturelle et la question du suicide assisté.

\section{DÉFINITION DE L'EUTHANASIE}

\section{ET PRINCIPES FONDATEURS}

Comme dit plus haut, l'article 2 de la loi reprend la définition proposée par le Comité consultatif de bioéthique : il y a lieu d'entendre par euthanasie l'acte pratiqué par un tiers qui met intentionnellement fin à la vie d'une personne à la demande de celle-ci. Ne sont pas considérés comme des euthanasies le fait de ne pas entamer ou de cesser des traitements ou encore d'administrer des analgésiques pour combattre la douleur, actes qui relèvent de la pratique médicale.

La loi relative à l'euthanasie présente des caractères hybrides: reprise dans les codes dans la partie civile, au chapitre du droit médical ${ }^{6}$, elle présente aussi des aspects pénaux puisqu'elle permet de sortir du champ infractionnel l'euthanasie pratiquée par un médecin se conformant aux conditions et procédure précisées. Plutôt que de parler de légalisation de l'euthanasie, il convient de décrire cette loi comme étant une dépénalisation conditionnelle.

Si le respect de l'autonomie de la personne humaine fonde la loi du 28 mai 2002 , il convient de souligner qu'en l'espèce, le principe de l'autonomie humaine n'est pas absolu. Il ne suffit pas que le patient formule une demande d'euthanasie : il faut encore que le médecin arrive à la conclusion, avec le patient, que les conditions posées par la loi sont remplies.

CONDITIONS ESSENTIELLES (ART. $3 \S 1^{\mathrm{er}}$ )

L'acte d'euthanasie doit être posé par un médecin: celui-ci ne peut absolument pas déléguer cette responsabilité, par exemple à un infirmier.

Le médecin doit s'assurer que sont réunies les trois conditions essentielles suivantes :

1. la demande est volontaire, réfléchie et réitérée, formulée indépendamment de toute pression extérieure; cette 
demande doit émaner d'un patient compétent, c'est-à-dire majeur et lucide;

2. le patient doit se trouver dans une situation médicale sans issue, et ce, à la suite d'une affection accidentelle ou pathologique grave et incurable;

3. le patient doit faire état d'une souffrance physique ou psychique insupportable.

Explicitons ces conditions.

\section{Première condition :}

la demande du patient

La demande du patient est au cœur de cette loi: sans demande du patient, il ne peut être question d'euthanasie. Au regard de cette loi, il ne peut donc être question de qualifier d'euthanasie une demande qui émanerait, par exemple, d'un membre de la famille ou de toute autre personne que le patient.

Le législateur belge a exclu la possibilité pour des mineurs d'accéder à une demande d'euthanasie, sauf pour les mineurs émancipés, catégorie fort marginale. Outre l'absence de consensus concernant ce point lors des débats parlementaires, la loi relative à l'euthanasie étant basée sur le principe de l'autonomie du patient, il eût été difficile d'inclure les mineurs d'âge. Il faut cependant remarquer que la loi sur les droits du patient du 22 août 2002 a introduit une notion différente de majorité «médicale»: le patient mineur estimé apte à apprécier raisonnablement ses intérêts peut en effet refuser un traitement même si ce refus entraîne l'abréviation de sa vie (article 12 § 2).

La loi relative à l'euthanasie insiste sur le nécessaire caractère volontaire et réfléchi de la demande du patient:

Article $3 \S 1^{\mathrm{er}}$ : «la demande est

formulée de manière volontaire, réfléchie et répétée» «elle ne résulte

pas d'une pression extérieure»;

Article $3 \S 2$ : «s'assurer [...] de sa

volonté réitérée»

Article $3 \S 1^{\text {er }}$ «...caractère volontaire, répété, réfléchi de la demande».

La préoccupation du législateur est très claire: il ne s'agit en aucun cas d'ouvrir l'euthanasie à des demandes suscitées par un découragement temporaire, ou induites par des considérations extérieures.

Le patient doit aussi être capable et conscient lorsqu'il formule sa demande. La question de la capacité doit être examinée, non pas dans le sens juridique strict du concept mais bien en ce qui concerne les capacités de compréhension par le patient de sa situation médicale et des enjeux qui se posent: il est non seulement question de souffrance mais aussi et essentiellement de vie et de mort. Autrement dit, une personne placée sous administration provisoire de ses biens parce qu'elle souffrirait, par exemple, de la maladie d'Alzheimer peut parfaitement être en mesure de prendre des décisions qui concernent son intégrité physique, en ce compris formuler une demande d'euthanasie.

Soulignons que la loi ouvre un droit à demander l'euthanasie et non un droit à l'euthanasie!

\section{Deuxième condition:}

une souffrance physique

ou psychique insupportable

Cette condition est éminemment subjective: qui pourrait être mieux placé que le patient pour juger du caractère insupportable de sa souffrance? Mais celle-ci est estimée en dialogue avec le médecin dont la première mission, après avoir écouté son patient, est de tenter de répondre à ses plaintes.

Les parlementaires étaient divisés en ce qui concerne l'adjonction des souffrances psychiques aux souffrances physiques. La loi a tranché en reconnaissant que la souffrance peut donc être analysée tant du point de vue de la douleur physique que du point de vue de la souffrance morale. En effet, il est parfois possible de contrôler les douleurs physiques tout en restant impuissant face aux souffrances morales: désespérance, perte de la dignité, sentiment de perdre toute autonomie de mouvement. Le législateur a tenu compte de cette dernière hypothèse: l'impossibilité d'apporter une réponse adéquate à la souffrance morale même si les douleurs physiques sont contrôlées.

\section{Troisième condition:}

\section{une affection grave et incurable}

Le législateur n'a pas donné de liste des affections graves et incurables qui peuvent donner lieu à une demande d'euthanasie. Chaque cas est unique: une personne atteinte de la sclérose en plaques pourra vouloir aller jusqu'au bout du chemin sans souhaiter que celui-ci soit abrégé. Donner une liste aurait pu constituer une pression et ne cadre pas avec cette volonté du législateur de respecter le choix de toute personne.

La loi parle de «situation médicale sans issue». Ceci ne peut en aucun cas être assimilé à la phase terminale. En effet, Le législateur s'est refusé à utiliser les concepts de phase terminale ou de patient terminal. Le débat a certes été profond à cet égard. Jusqu'à la dernière minute, des amendements ont été proposés pour introduire la notion de phase terminale.

En revanche, il est prévu des conditions supplémentaires dans l'hypothèse où le médecin estime que le décès n'est pas prévisible à bref délai (voir infra).

L'affection médicale peut résulter soit d'un accident, soit d'une pathologie.

\section{CONDITIONS DE FORME}

ET DE PROCÉDURE (ARTICLE 3 § 2)

Afin de s'assurer du respect des conditions essentielles de la loi, le législateur belge a prévu une série de balises: demande écrite, devoir d'information du médecin traitant, consultation d'un deuxième, voire d'un troisième médecin, conditions supplémentaires à respecter lorsque le décès n'est pas prévisible à brève échéance, entretien avec l'équipe soignante, un entretien éventuel avec les proches, la consignation de tous les éléments dans le dossier médical.

\section{Demande écrite du patient}

La demande doit être formulée par écrit et, dans l'hypothèse où le patient n'est pas en état de le faire lui-même, cette demande doit être écrite par un tiers qui ne peut avoir un intérêt matériel au décès du patient, et ce, en présence du médecin.

\section{Devoir d'information}

du médecin traitant

Le médecin saisi d'une demande doit avoir au préalable informé le patient de son état de santé, de son espérance de vie, des possibilités de traitements, des soins palliatifs accessibles. Il s'agit donc d'une obligation d'information concernant les soins palliatifs et non de filtre palliatif. Il ne peut dès lors être question ni d'imposer au patient une consultation auprès d'un médecin spécialisé en soins palliatifs, ni de le soumettre à des soins palliatifs afin que sa demande soit recevable. Ceci doit être mis en rapport avec la loi sur les droits du patient qui permet à ce dernier de refuser un traitement, quel qu'il soit. La même loi sur les droits du patient exige aussi une information claire, intelligible pour le patient.

La loi relative à l'euthanasie exige également que le médecin conduise plusieurs entretiens avec son patient pour s'assurer de la persistance des intentions de celuici : à tout instant, le patient peut renoncer à sa demande. Et cette demande ne peut résulter que d'un choix, après avoir reçu toute l'information nécessaire.

\section{Le rôle des médecins consultants}

L'intervention d'un médecin consultant, avec un regard nouveau, se révèle particulièrement utile dans l'hypothèse d'une relation fusionnelle du médecin traitant avec son patient qu'il aura peut-être suivi depuis des années, dès le diagnostic d'une maladie grave et incurable. 
Le médecin consultant doit être indépendant par rapport à la relation thérapeutique qui s'est nouée entre le patient et le médecin recevant la demande d'euthanasie; il doit aussi être compétent quant à la pathologie concernée. Il doit non seulement consulter le rapport médical mais également examiner le patient afin de vérifier d'une part le caractère grave et incurable de l'affection et d'autre part s'assurer du caractère constant, insupportable et inapaisable de la souffrance physique ou psychique.

Il est toutefois rappelé que la souffrance physique ou psychique est un élément de nature éminemment subjective: le patient est en principe le mieux placé pour en estimer le caractère insupportable ${ }^{7}$. Mais ce caractère subjectif est tempéré par le regard de professionnels, le médecin traitant et le médecin consultant qui sont nécessairement confrontés à cette question: n'est-il pas possible d'apaiser cette souffrance, de proposer un traitement pour combattre la douleur ou pour rencontrer la souffrance psychique?

Le cas du décès non prévisible à brève échéance requiert l'intervention d'un troisième médecin, soit un deuxième médecin consultant, comme on le verra ci-dessous.

\section{Décès non prévisible à brève échéance}

Des conditions plus strictes sont prévues dans l'hypothèse où le médecin estime que le décès n'est pas prévisible à brève échéance.

Le législateur poursuit deux objectifs en mentionnant cette hypothèse: d'une part, éviter le piège de la définition de phase terminale ou de patient terminal, et d'autre part se donner plus de temps et offrir plus de garanties lorsque le décès peut encore être considéré comme éloigné. La Commission fédérale de contrôle et d'évaluation estime que quand le décès est attendu dans les jours, les semaines ou les mois qui viennent, il peut être considéré comme prévisible à brève échéance.

En cas de décès non prévisible à brève échéance, il faudra respecter un délai d'au minimum un mois entre la demande écrite et l'acte d'euthanasie. Un troisième médecin devra être consulté, qui devra être spécialiste de la pathologie concernée ou encore psychiatre et dont la mission sera d'examiner tout particulièrement le caractère volontaire de la demande et l'existence d'une souffrance inapaisable.

\section{Entretien avec l'équipe soignante}

S'il existe une équipe de soins, le médecin devra s'entretenir avec elle ou certains de ses membres afin de recueillir son avis. Toutefois, l'équipe n'a aucun pouvoir décisionnel.

\section{Entretien avec les proches}

La loi prévoit que le médecin s'entretiendra de sa demande avec les proches du patient si telle est sa volonté.

\section{Dossier médical}

Tous les éléments en relation avec le processus d'euthanasie doivent être consignés au dossier médical.

\section{DÉCLARATION ANTICIPÉE D'EUTHANASIE}

(ARTICLE 4)

Cet aspect de la loi a été fortement débattu. Parmi les parlementaires qui étaient certes favorables au principe de la dépénalisation de l'euthanasie pour des patients conscients, certains se posaient la question de l'opportunité d'un document écrit avant même de se trouver dans les conditions d'affection grave et incurable et de souffrance insupportable. Et si l'on changeait d'avis?

Le législateur s'est donc montré très restrictif et formaliste pour la déclaration anticipée que toute personne adulte ou tout mineur émancipé peut écrire. Cette déclaration doit être signée par deux témoins dont l'un au moins ne peut avoir d'intérêt matériel au décès du déclarant. Elle doit avoir été rédigée dans les cinq ans qui précèdent l'impossibilité pour le patient de manifester sa volonté. Elle peut être retirée ou adaptée à tout moment.

Le médecin ne pourra s'appuyer sur une telle déclaration que pour autant que le déclarant se trouve en état d'inconscience irréversible selon l'état actuel de la science.

Le déclarant peut désigner une ou des personnes de confiance qui seront son ou ses porte-parole lorsqu'il ne pourra plus s'exprimer. Il ne s'agit donc pas de mandataires: tout comme la demande en cas de conscience du patient, la déclaration anticipée n'a pas de force contraignante et le médecin peut refuser de pratiquer une euthanasie (voir infra: Liberté de conscience).

Le médecin devra par ailleurs respecter les conditions de forme et de procédure suivantes: consultation d'un second médecin qui examinera le dossier médical et le patient pour se prononcer sur le caractère irréversible de l'inconscience, entretien avec l'éventuelle équipe soignante, entretien avec l'éventuelle personne de confiance, entretien avec les proches désignés par l'éventuelle personne de confiance.

Depuis le $1^{\text {er }}$ septembre 2008, il est prévu une possibilité d'enregistrement des déclarations anticipées via les Communes afin de constituer auprès du Service public fédéral de la santé une banque de données accessible 24 h sur 24 aux médecins ${ }^{8}$.
LE CONTRÔLE SOCIÉTAL DE LA LOI: PROCÉDURE ET COMPOSITION DE LA COMMISSION DE CONTRÔLE ET D'ÉVALUATION

Pour cet aspect de la loi, je renvoie à la contribution du professeur Fernand Van Neste dans ce numéro et ne reprends les éléments de la loi que de façon lapidaire.

Dans les quatre jours ouvrables de l'acte d'euthanasie, le médecin doit en faire la déclaration auprès de la Commission fédérale de contrôle et d'évaluation de l'application de la loi relative à l'euthanasie.

Cette commission est composée de 16 membres: 8 médecins, 4 juristes, 4 membres issus des milieux concernés par la problématique de patients atteints de maladie incurable. La parité linguistique doit être respectée ainsi qu'une représentation pluraliste: la commission comporte des membres qui ne sont pas nécessairement favorables à la dépénalisation de l'euthanasie.

Le premier objet de cette commission est d'assurer le contrôle par la société des actes d'euthanasie. Elle a donc à examiner, sur base des déclarations, le respect par les médecins des conditions prévues par la loi. A priori, l'anonymat de tous les intervenants est préservé. Ce n'est qu'en cas de doute et à la suite d'un vote à la majorité simple que la commission peut décider de lever l'anonymat. Dans l'hypothèse où la commission estime que les conditions essentielles de la loi n'ont pas été respectées, par un vote à la majorité des deux tiers, le dossier est transféré au procureur du Roi.

La Commission a par ailleurs une mission d'évaluation et établit tous les deux ans un rapport concernant l'application de la loi destiné au Parlement.

RÔLE DES PHARMACIENS (ARTICLE 3 BIS)

Depuis le vote de la loi en 2002, une seule modification a été apportée au texte initial, et ce, à la demande expresse des pharmaciens. Par la loi du 10 novembre 2005, un article 3 bis a été inséré dans la loi du 28 mai 2002. Il précise que le pharmacien ne commet aucune infraction s'il délivre les médicaments indiqués pour une euthanasie. Il appartient au médecin de tracer la prescription en précisant que ces médicaments seront utilisés dans le cadre d'une euthanasie. Ces médicaments seront remis par le pharmacien au médecin en personne. Il incombe aux autorités publiques de prendre les mesures nécessaires pour assurer la disponibilité des substances nécessaires, y compris dans les pharmacies hors hôpital. 


\section{LIBERTÉ DE CONSCIENCE (ARTICLE 14)}

La loi, qui se fonde sur le respect de l'autonomie et de l'intégrité physique de la personne désireuse d'accéder à l'euthanasie, devait prévoir ce même respect dans le chef du médecin et de toute autre personne qui serait amenée à intervenir d'une manière ou d'une autre dans un processus d'euthanasie.

L'article 14 de la loi prévoit qu'aucun médecin n'est tenu de pratiquer une euthanasie et qu'aucune personne n'est tenue de participer à une euthanasie.

Cela étant, si le médecin consulté refuse pour une raison personnelle de respect de ses convictions ou pour tout motif, il est tenu d'en informer en temps utile son patient ou la personne de confiance éventuelle, tout en précisant les raisons, et à la demande du patient ou de la personne de confiance, il doit communiquer le dossier médical à un autre médecin désigné par le patient ou la personne de confiance. Soulignons d'abord ce qu'implique ce deuxième aspect: il n'y a pas une obligation de transfert automatique du dossier médical. Ceci peut poser problème car le patient ou la personne de confiance ne connaissent pas nécessairement le nom d'un médecin qui pourrait entendre la demande d'euthanasie. Ceci implique également que le processus devra être repris $a b$ initio: le nouveau médecin consulté devra en effet construire une relation thérapeutique profonde avec son patient avant de pouvoir répondre à une demande d'euthanasie. Quant au premier aspect, si le médecin doit communiquer les raisons de son refus, il n'a pas pour autant à se justifier: il est libre de refuser de pratiquer un tel acte. Mais le patient doit connaître la teneur des raisons qui motivent ce refus. Est-ce au nom d'une conception philosophique ou religieuse? En ce cas, il est impérieux que le patient s'adresse à un autre médecin sans autre délai. Est-ce parce que le médecin se sent insuffisamment formé et informé? Dans cette hypothèse, il serait possible de faire appel à un médecin apte à répondre aux questionnements de son confrère dont le refus s'estompera peut-être, la relation thérapeutique pouvant alors se poursuivre en toute confiance. Une autre hypothèse serait que le médecin traitant estime que le cas n'entre pas dans le champ d'application de la loi. Il est alors utile que le patient le sache, afin de pouvoir lui-même prendre sa décision: soit se rendre à l'avis de son médecin traitant, soit consulter un autre médecin qui, à la lumière du dossier médical transmis par son confrère, connaîtra déjà les diagnostic et pronostic.

Cette clause de conscience donne lieu à controverse dans son application. Est-elle l'apanage du seul médecin, personne physique? Peut-elle être invoquée par une institution qui interdirait à ses médecins de pratiquer une euthanasie? Une institution peut-elle refuser un patient parce qu'il est porteur d'une demande d'euthanasie? Pourrait-elle opposer un simple refus à la demande d'un patient sans lui permettre d'être en mesure de voir respecter à tout le moins l'écoute de sa demande? Sans que la loi du 28 mai 2002 ait institué un droit à l'euthanasie, il paraît à tout le moins qu'elle impose l'obligation d'une réponse à une requête d'euthanasie, et non un simple déni. Par ailleurs, il semble difficile de transposer la clause de conscience, qui est une prérogative individuelle, à une institution.

\section{MORT NATURELLE (ARTICLE 15)}

La personne décédée à la suite d'une euthanasie légale est réputée décédée de mort naturelle, notamment pour ce qui concerne les contrats d'assurance-vie. Il en est de même pour les actes de décès.

\section{EUTHANASIE ET SUICIDE ASSISTÉ}

La loi du 28 mai 2002 n'a pas précisé la méthode à suivre pour pratiquer l'euthanasie: c'est donc en concertation avec le patient, selon l'état et les volontés de ce dernier, que le médecin optera pour la perfusion ou pour la potion létale. À condition que le médecin observe tous les prescrits de la loi - notamment son obligation d'assister son patient jusqu'au dernier souffle -, l'acte posé par le patient de boire la solution de barbituriques qui lui est donnée par le médecin sera assimilé à une euthanasie ${ }^{9}$.

En revanche, si le médecin se contente de prescrire les médicaments nécessaires, sans être présent au moment de l'acte, ce comportement relèvera du champ pénal.

\section{LE CODE DE DÉONTOLOGIE ACTUEL}

L'Ordre des médecins a procédé à une révision de son Code de déontologie en ce qui concerne la vie finissante le 18 mars 2006. L'interdit de l'euthanasie et celui du suicide assisté ont désormais disparu. Conformément à l'article $95 \$ 2$ du Code actuel, lors de toute demande à propos de la fin de vie, le médecin est tenu d'expliquer les initiatives qui peuvent être prises telles que la désignation d'un mandataire (en conformité avec la loi sur les droits du patient du 22 août 2002), la consignation du refus de consentement à une intervention déterminée et la rédaction d'une déclaration anticipée concernant l'euthanasie. Le paragraphe 4 de l'article 97 prévoit que le médecin aide le patient dans la rédaction et la conservation des déclarations définies à l'article $95 \$ 2$.
Avant cette réforme de 2006, l'Ordre des médecins avait publié le 22 mars 2003 un avis fondamental portant sur les lois relatives à l'euthanasie, aux soins palliatifs et aux droits du patient. L'Ordre y précisait qu'un médecin ne pourrait faire l'objet d'une sanction sur base de l'article 95 non encore modifié s'il s'est conformé aux prescrits d'une législation à portée éthique établie dans un état démocratique et respectant la liberté de conscience du médecin. Il est également question du devoir d'information claire et en temps utile qui pèse sur le médecin. Dans sa conclusion, l'Ordre insiste sur «l'essence des dispositions légales analysées, à savoir le respect mutuel de l'autonomie et des valeurs éthiques de chacun» ainsi que sur une compréhension réciproque. «Un patient dûment informé pourra faire en concertation franche avec son médecin son choix de vie».

$* * *$

Certes, l'euthanasie reste un domaine en débat dans la société belge. Des propositions de loi sont déposées à chaque législative en vue d'étendre le champ d'application de la loi aux mineurs d'âge et aux patients atteints de démences séniles dégénératives telles que la maladie d'Alzheimer, dans l'hypothèse où ils ne sont plus capables d'exprimer valablement leurs volontés. La clause de conscience et son application posent problème. La question controversée de la déclaration anticipée a donné lieu à une législation qui est en définitive très formaliste et contraignante, de telle sorte que de nombreuses personnes renoncent à remplir cet outil pourtant bien utile.

La loi adoptée par le Parlement belge en 2002 représente une solution qui tient compte des conceptions éthiques alors existantes dans la société belge mais elle ne peut être considérée comme LA solution, ainsi que l'exprimait le parlementaire Fred Erdman à la clôture des débats devant la Chambre des représentants. Cette législation, nécessairement imparfaite puisque œuvre humaine, a eu le mérite de briser le tabou de la mort choisie en proposant la dépénalisation conditionnelle de l'euthanasie. Et il convient de constater que le débat en lui-même s'est apaisé et qu'aucune dérive annoncée n'a été constatée.

Il faut rappeler que nul ne peut être contraint ni à demander l'euthanasie, ni à la pratiquer ou encore à y participer d'une façon quelconque. La loi belge propose un cadre qui laisse à tout un chacun sa responsabilité. Celle du patient de demander ou non l'euthanasie, d'en parler avec son médecin, voire avec ses proches, de 
poursuivre sa lutte contre la maladie, de bénéficier de soins palliatifs et peut-être un jour de préciser la date, le lieu et le comment de sa mort assistée par son médecin. Celle du médecin traitant qui se doit d'entendre la requête de son patient, de lui répondre en toute franchise de sa volonté ou non d'entamer ce chemin qui pourrait le conduire à pratiquer une euthanasie, de discuter avec son patient de toutes les ouvertures possibles et peut-être un jour, de convenir avec lui de la date, du lieu où prendra place cet acte ultime.

Il ne s'agit nullement de faire l'apologie de cette législation, nécessairement perfectible. Son application dépend de ce que nous, femmes et hommes, patients et médecins, famille et personnel paramédical, en ferons. C'est notre responsabilité de rester en perpétuel questionnement et de ne pas apporter de réponses toutes faites à des questions existentielles. Et il faut surtout se rappeler qu'il est question de choix, de responsabilité et aussi de solidarité et non d'une décision qui serait induite par des considérations extérieures et économiques.

\section{Bibliographie}

CASSIERS, L., Y. ENGLERT, A. VAN ORSHOVEN et E. VERMEERSCH (dir.) (2001). Les avis du Comité consultatif de Bioéthique de Belgique: 1996-2000, Bruxelles, De Boeck Université.

HERREMANS, J. (2003). "La chambre du conseil de Liège applique la loi du 28 mai 2002 sur l'euthanasie», dans Journal des procès, liv. 455, 31. Sommaire Commentaire de Corr. Liège (Ch. cons.) 6 février 2003.

HERREMANS, J. et Y.-H. LELEU (2003). "De la clause de conscience en matière d'euthanasie », Le Soir, 2003-06-06.

LALLEMAND, R. (2000). "L'homme face à la souffrance et la mort», dans Le songe du politique, avant-propos de Régis Debray, Bruxelles, Éd. Didier Devillez.

LEENEN, H.J.J. (1985). "Euthanasie voor de Hoge Raad», dans Nederlands Tijdschrift voor Geneeskunde, p. 414-417.

LELEU, Y.-H. et G. GENICOT (2004). "L'euthanasie en Belgique et aux Pays-Bas », Revue trimestrielle des droits de l'homme, liv. 57, 5-50.

RIGAUX, F. (2002). "L'avis du Conseil d'État belge sur la dépénalisation de l'euthanasie et le droit aux soins palliatifs", Revue trimestrielle des droits de l'homme, p. 274-285.

\section{Notes}

1. Loi du 28 mai 2002 relative à l'euthanasie (publiée au Moniteur belge le 22 juin 2002 et entrée en vigueur le 22 septembre 2002); loi du 14 juin 2002 relative aux soins palliatifs (Moniteur belge: 26 octobre 2002); loi du 22 août 2002 relative aux droits du patient (Moniteur belge: 26 septembre 2002).

2. Loi du 12 avril 2001 relative au contrôle de l'interruption de vie sur demande et du suicide assisté - WET van 12 april 2001, houdende toetsing van levensbeëindiging op verzoek en hulp bij zelfdoding.

3. Voir le site Internet de l'Ordre des médecins de Belgique: <http;//www.ordomedic.be/fr/ code/contenu/>.

4. Voir Cassiers, Englert, Van Orshoven et Vermeersch (dir.), 2001, p. 11-15. Les avis du Comité consultatif de bioéthique de Belgique peuvent aussi être consultés sur le site Internet <http://www.sante.belgique.be/bioeth>.

5. Roger Lallemand, ministre d'État, avocat au barreau de Bruxelles, grand humaniste, coauteur de la loi relative à l'interruption de grossesse, auteur d'une loi bannissant les mines antipersonnel servant de modèle pour le Traité d'Ottawa; à lire sa réflexion sur "L'homme face à la souffrance et la mort » (Lallemand, 2000 ; il existe diverses publications de ce texte).

6. Sommaire du droit médical : droits du patient, en ce compris la loi sur les soins palliatifs, recherche sur embryons, euthanasie, expérimentations sur la personne humaine, médicaments, prélèvement et transplantation d'organes et sang humain.

7. Voir la brochure éditée par la Commission fédérale de contrôle et d'évaluation de la loi à l'intention des médecins ainsi que les autres documents (loi, arrêtés, royaux, formulaires de déclaration) sur le site Internet de la Commission hébergé par le site du Service public fédéral de la Santé publique: <http://www. health.belgium.be>.

8. 27 avril 2007. Arrêté royal réglant la façon dont la déclaration anticipée en matière d'euthanasie est enregistrée et est communiquée via les services du Registre national aux médecins concernés, Moniteur belge, 7 juin 2007.

9. C'est en tout cas l'interprétation tant du conseil de l'Ordre des médecins que de la commission d'évaluation et de contrôle de l'euthanasie. 